\title{
Dissertações e teses sobre cuidados paliativos em oncologia pediátrica: estudo bibliométrico
}

\author{
Dissertations and theses on palliative care in pediatric oncology: a bibliometric study
} Tesis de maestría y doctorado sobre cuidados paliativos en oncología pediátrica: estudio bibliométrico

Kalina Coeli Costa de Oliveira Dias ${ }^{1}$ iD https://orcid.org/0000-0001-9076-2575

Patrícia Serpa de Souza Batista ${ }^{1}$ ic https://orcid.org/0000-0001-5080-1605

Maria Andréa Fernandes ${ }^{1}$ io https://orcid.org/0000-0002-1873-5385

Ana Aline Lacet Zaccara ${ }^{1}$ ic https://orcid.org/0000-0002-5222-2049

Thaís Costa de Oliveira ${ }^{1}$ id https://orcid.org/0000-0003-1728-1905

Monica Ferreira de Vasconcelos ${ }^{1}$ id https://orcid.org/0000-0002-7856-1890

Amanda Maritsa de Magalhães Oliveira ${ }^{1}$ io https://orcid.org/0000-0002-4709-1742

Fernanda Ferreira de Andrade² id https://orcid.org/0000-0002-0918-2512

Dias KC, Batista PS, Fernandes Como citar:

AA, Oliveira TC, Vasconcelos MF, et al. Dissertações e teses sobre cuidados paliativos em oncologia pediátrica: estudo bibliométrico. Acta Paul Enferm. 2020;33:APE20190264.

DOI

http://dx.doi.org/10.37689/actaape/2020A002642

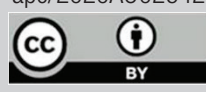

cologia: Pediatria; Bibliometria; Dissertação acadêmica

Keywords Paliative care; Oncology; Pediatrics; Bibliometrics; Academmic dissertations

Descriptores

Cuidados paliativos; Oncología; Pediatría; Bibliometría; Tesis académica

Submetido
10 de Setembro de 2019
Aceito
28 de Janeiro de 2020

Autor correspondente

Kalina Coeli Costa de Oliveira Dias E-mail: kalinacoeli@gmail.com

\section{Resumo}

Objetivo: Analisar indicadores bibliométricos de estudos oriundos de dissertações e teses sobre cuidados paliativos em oncologia pediátrica defendidas em Programas de Pós-Graduação no cenário brasileiro.

Métodos: Estudo bibliométrico realizado por meio do Banco de Teses e Dissertações da Coordenação de Aperfeiçoamento de Pessoal de Nível Superior (CAPES) e da Biblioteca Digital Brasileira de Teses e Dissertações (BDTD), entre os anos de 2008 a 2018, defendidas em Programas de Pós-Graduação do Brasil.

Resultados: Os indicadores bibliométricos investigados destacam 60 estudos (13 teses e 47 dissertações) realizados sobre cuidados paliativos em oncologia pediátrica. A Instituição de Ensino Superior com a maior produção científica foi a Universidade de São Paulo, e a Região Sudeste foi a que mais se destacou com o maior quantitativo de publicações; estas foram distribuídas em 18 Programas. A área da Enfermagem ocupou posição de destaque seguida da Psicologia e da Medicina. 0 desenho metodológico mais utilizado nos estudos foi de natureza qualitativa. A maioria das pesquisas foi realizada em hospitais especializados para 0 tratamento de câncer.

Conclusão: A pesquisa identificou um número reduzido de estudos oriundos de dissertações e teses sobre cuidados paliativos em oncologia pediátrica, defendidas em Programas de Pós-Graduação no cenário brasileiro. Sugerem-se novos estudos para ampliar a produção científica acerca do tema bem como para disseminar evidências científicas no contexto da prática clínica dos cuidados paliativos em oncologia pediátrica.

\section{Abstract}

Objective: To analyze bibliometric indicators of studies originated from dissertations and theses on palliative care in pediatric oncology defended in Postgraduate Programs in the Brazilian scenario.

Methods: Bibliometric study conducted through the Bank of Theses and Dissertations of the Coordination for the Improvement of Higher Education Personnel (Portuguese acronym: CAPES) and the Brazilian Digital Library of Theses and Dissertations (Portuguese acronym: BDTD) between years 2008 and 2018 defended in Postgraduate Programs in Brazil.

Results: The investigated bibliometric indicators highlighted 60 studies (13 theses and 47 dissertations) conducted on palliative care in pediatric oncology. The Higher Education Institution with the highest scientific production was the Universidade de São Paulo. The southeast region stood out with the largest number of publications distributed in 18 programs. The Nursing field occupied a prominent position, followed by Psychology and Medicine. The qualitative methodological design was the most used in the studies. Most studies were conducted in specialized hospitals for cancer treatment. 
Conclusion: A small number of studies originating from dissertations and theses on palliative care in pediatric oncology defended in Postgraduate Programs in the Brazilian scenario was identified in this investigation. Further studies are suggested to expand scientific production on the topic and disseminate scientific evidence within clinical practice of palliative care in pediatric oncology.

\section{Resumen}

Objetivo: Analizar indicadores bibliométricos de estudios oriundos de tesis de maestría y doctorado sobre cuidados paliativos en oncología pediátrica defendidas en Programas de Posgrado en el escenario brasileño.

Métodos: Estudio bibliométrico realizado por medio del Banco de Teses e Dissertações de la Coordenação de Aperfeiçoamento de Pessoal de Nível Superior (CAPES) y de la Biblioteca Digital Brasileira de Teses e Dissertações (BDTD), entre los años 2008 y 2018, defendidas en Programas de Posgrado de Brasil.

Resultados: Los indicadores bibliométricos investigados destacan 60 estudios (13 tesis de doctorado y 47 tesis de maestría) realizados sobre cuidados paliativos en oncología pediátrica. La institución de educación universitaria con mayor producción científica fue la Universidad de São Paulo, y la región sudeste fue la que más se destacó con el mayor cuantitativo de publicaciones; estas se distribuyeron en 18 Programas. El área de Enfermería ocupó una posición destacada, seguida de Psicología y Medicina. El diseño metodológico más utilizado en los estudios fue de naturaleza cualitativa. La mayoría de las investigaciones fue realizada en hospitales especializados en tratamiento de cáncer.

Conclusión: La investigación identificó un número reducido de estudios oriundos de tesis de maestría y doctorado sobre cuidados paliativos en oncología pediátrica, defendidas en Programas de Posgrado en el escenario brasileño. Se sugieren nuevos estudios para ampliar la producción científica sobre el tema, así como también para difundir evidencias científicas en el contexto de la práctica clínica de los cuidados paliativos en oncología pediátrica.

\section{Introdução}

O câncer é a primeira causa de morte por doença na população infantojuvenil com idades entre $1 \mathrm{a}$ 19 anos, tanto no Brasil quanto em países desenvolvidos. Quando diagnosticado precocemente, a estimativa de cura é de até $80 \%$ dos casos. Em países desenvolvidos as taxas de mortalidade são menores em decorrência de melhores condiçóes no acesso aos serviços médicos, de diagnóstico e tratamento. No Brasil, essas melhorias ainda não foram percebidas na mesma proporção e há perceptível variação das taxas de cura de neoplasias em crianças e adolescentes variando de $50 \%$ na Região Norte, $60 \%$ na Região Nordeste, $65 \%$ na Região CentroOeste, $70 \%$ na Região Sudeste e 75\% na Região Sul. Quando as possibilidades de cura se esgotam, surgem como alternativa de assistência terapêutica especializada os cuidados paliativos. ${ }^{(1)}$

$\mathrm{Na}$ última década, os cuidados paliativos pediátricos no Brasil têm apresentado desenvolvimento exponencial por meio da implantação de políticas públicas no âmbito do Sistema Único de Saúde (SUS) com a criação de Política Nacional para a Prevenção e Controle do Câncer, em 2013, com a Portaria No 874 de 16 de maio de 2013 e da publicação das diretrizes para a organização dos cuidados paliativos ligados aos cuidados continuados integrados no SUS com Resoluçáo No 41 de 31 de outubro de 2018. ${ }^{(2)}$ Segundo o relatório publicado pela Academia Nacional de Cuidados Paliativos (ANCP), advindo da análise situacional e recomendaçóes para estruturação de programas de cuidados paliativos no Brasil, desperta-se a atenção para a precarização e para a desigualdade de disponibilidade desses cuidados no cenário nacional. Dos 177 centros de cuidados paliativos cadastrados no país, apenas $21 \%$ deles são voltados à assistência pediátrica, sendo mais de 50\% do total na Região Sudeste do país e concentrado na assistência hospitalar. ${ }^{(2)}$

Os cuidados paliativos no campo pediátrico são cada vez mais reconhecidos como parte integrante do cuidado de crianças com doenças que ameaçam a continuidade da vida. Conceitualmente, tais cuidados visam melhorar a qualidade de vida do paciente e de sua família durante toda a trajetória da doença e podem aliviar os sintomas de desconforto e estresse de crianças que vivem em condições de risco de vida e de suas famílias, permeados por práticas assistenciais específicas para redução de sintomas desagradáveis fornecidos por uma equipe multiprofissional voltada às dimensôes biopsicossociais e espirituais. ${ }^{(3)}$

Por essa razão, o aprimoramento dos cuidados paliativos pediátricos é uma necessidade nacional urgente, visto a relevância e o impacto positivo das medidas de cuidados paliativos na qualidade de vida de crianças doentes e de suas famílias. ${ }^{(4)}$ Portanto, é necessária uma maior disseminação do conhecimento produzido nos programas de pós-graduação do país acerca dos cuidados paliativos em oncologia pediátri- 
ca, por meio de estudos que busquem proporcionar uma maior visibilidade à produção científica proveniente de dissertaçóes e teses sobre a referida temática, por exemplo, a partir de pesquisas bibliométricas.

Reportando-se aos estudos bibliométricos, estes possibilitam o direcionamento de novas investigaçóes sobre o tema e contribuem para obtenção de indicadores da produção científica, identificando temporalidade, origem dos trabalhos, temas e metodologias empregadas. ${ }^{(5)}$ Considerando a importância de investigar indicadores bibliométricos que avaliem a atividade científica acerca da temática, é oportuno realizar um estudo cujo fio condutor parte da seguinte questáo norteadora: Qual a caracterização de dissertaçóes e de teses sobre cuidados paliativos em oncologia pediátrica defendidas em Programas de Pós-Graduação no Brasil? Para tanto, o estudo apresenta como objetivo: analisar indicadores bibliométricos de estudos oriundos de dissertaçóes e teses sobre cuidados paliativos em oncologia pediátrica defendidas em Programas de PósGraduação no cenário brasileiro.

\section{Métodos}

Para selecionar a amostra foram utilizados os termos "cuidados paliativos" and "criança" and "câncer". Em seguida, foram especificados os critérios de inclusão: publicaçôes nas modalidades de dissertaçóes e teses, divulgadas no idioma português e publicadas no período de janeiro de 2008 a dezembro de 2018. Por se tratar de uma pesquisa bibliométrica é importante um recorte temporal que contemple um maior número de publicaçóes de dissertaçóes e teses para ampliar a amostra e obter um quantitativo significativo de estudos.

$\mathrm{Na}$ segunda etapa foi realizada a coleta de dados. Para tanto, foi empregado um instrumento elaborado pelas autoras que tornasse possível contabilizar a atividade cientifica produzida e gerar indicadores bibliométricos. $\mathrm{O}$ instrumento contemplou as seguintes variáveis: título, modalidade de publicação, formação do pesquisador, nome da instituição, ano de defesa, regiáo, desenho metodológico, palavraschave e artigos originados das dissertaçóes e teses. Procedeu-se à coleta dos dados no período de março a julho de 2019. Os dados foram obtidos por meio da leitura dos resumos e, quando estes não apresentavam as informações necessárias, utilizou-se os textos na íntegra. Para a seleção dos estudos, seguiram-se as recomendaçóes do checklist do Statement for Reporting Systematic Review and Meta-Analyses of Studies - PRISMA, ${ }^{(5)}$ conforme a figura 1.

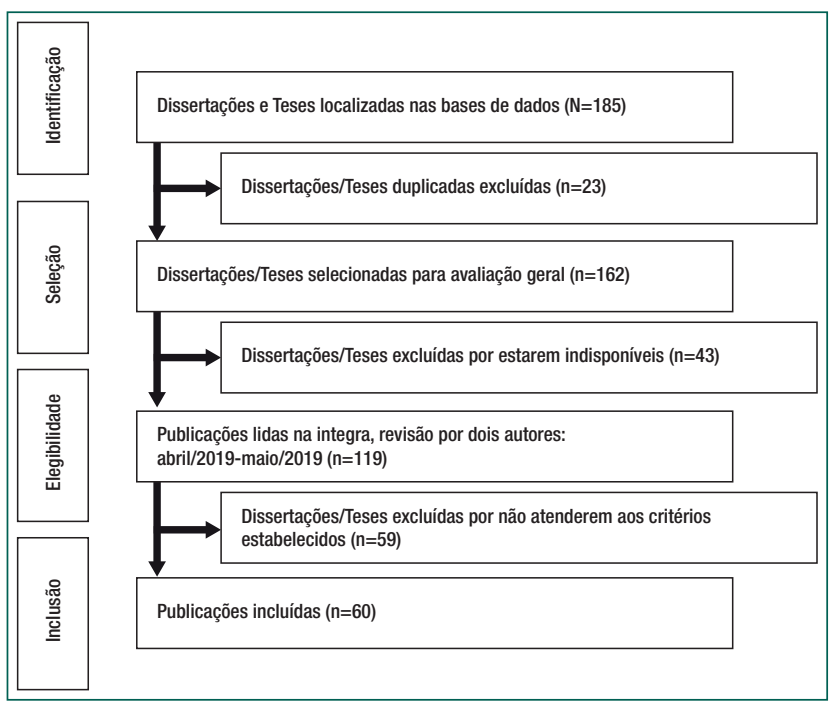

Figura 1. Fluxograma do processo de seleção dos estudos, adaptado do PRISMA

Os dados obtidos a partir das dissertações e teses selecionadas para o estudo foram extraídos com base no instrumento proposto para coleta dos dados. Estes foram apresentados por meio de representaçóes gráficas e analisados quantitativamente por meio de frequência absoluta e percentual por ferramenta do programa Microsoft Office Excel 2010.

Para organizar as palavras-chave, utilizou-se o mapa conceitual, uma ferramenta que adota a reprodução gráfica de conceitos relacionados e ligados através de proposiçóes e de palavras-chaves. Nas pesquisas de Enfermagem, os mapas conceituais são empregados com o intuito de estimular a reflexão por meio do pensamento crítico e de contribuir para solucionar problemas e sintetizar conceitos, no plano do cuidado, e avaliar açóes de enfermagem. ${ }^{(6)}$

Neste estudo, o mapa conceitual serviu para demonstrar a associação temática conceitual entre as palavras-chaves mencionadas nas dissertaçóes e nas teses selecionadas e o contexto do estudo. As palavras-chave foram agrupadas a partir das principais - 
Cuidados Paliativos e Oncologia Pediátrica - por serem o tema do estudo, e organizadas ordenadamente em classes temáticas, mas não de forma hierárquica.

\section{Resultados}

No recorte temporal compreendido entre 2008 e 2018, identificaram-se 60 produçóes científicas de dissertaçóes e teses sobre cuidados paliativos voltados para a criança com câncer, sendo 47 (78 \%) dissertaçôes de mestrado e 13 (22\%) teses de doutorado. O maior quantitativo de achados está entre os anos de 2011 e 2014, com um comportamento relativamente estável, sendo 2014 o ano de maior achado de dissertaçôes (9\%) e teses (23\%). Quanto à região geográfica da instituiçáo à qual os pesquisadores estavam vinculados, constatou-se que a Região Sudeste apresentou 36 (60\%) produçôes de teses e dissertações distribuídas em 18 Programas de Pós-Graduação. Seguindo esse padrão, destacou-se a Região Nordeste com 14 trabalhos $(23, \%)$ distribuídos em 9 Programas de Pós-Graduação, conforme demonstrado na tabela 1 em números absolutos e percentuais.

Tabela 1. Distribuição dos estudos acerca dos cuidados paliativos em oncologia pediátrica em relação à região, instituição de ensino superior, programas de pós-graduação, dissertações e teses $(n=60)$

\begin{tabular}{|c|c|c|c|c|c|}
\hline Região & Instituição & $\begin{array}{l}\text { Programas de } \\
\text { Pós-Graduação }\end{array}$ & $\begin{array}{c}\text { Dissertações } \\
\mathrm{n}\end{array}$ & $\begin{array}{c}\text { Teses } \\
\mathrm{n}\end{array}$ & $\begin{array}{c}\text { Dissertações/ } \\
\text { Teses } \\
\mathrm{n}(\%) \\
\end{array}$ \\
\hline \multirow[t]{7}{*}{ Nordeste } & Universidade Católica de Recife & Ciências da Linguagem & 1 & - & 1(2) \\
\hline & & Saúde Coletiva & & & \\
\hline & Universidade Federal do Maranhão & Saúde Materno-Infantil & 1 & - & $1(2)$ \\
\hline & Universidade Federal da Paraíba & Enfermagem & 4 & 2 & $6(10)$ \\
\hline & Universidade Federal do Rio Grande do Norte & Enfermagem & 2 & - & 2(2) \\
\hline & & Psicologia & & & \\
\hline & Universidade Federal de Pernambuco & Saúde da Criança e do Adolescente & - & 1 & $1(2)$ \\
\hline Norte & Universidade Federal do Pará & Psicologia & 1 & - & $1(2)$ \\
\hline \multirow[t]{13}{*}{ Sudeste } & Universidade de São Paulo - Ribeirão Preto & Psicologia & 11 & 3 & $14(23)$ \\
\hline & & Enfermagem em Saúde Pública & & & \\
\hline & & Neurologia & & & \\
\hline & Universidade Estadual do Rio de Janeiro & Enfermagem & 2 & - & $2(3)$ \\
\hline & & Saúde Coletiva & & & \\
\hline & Universidade Federal do Rio de Janeiro & Enfermagem & 1 & - & $1(2)$ \\
\hline & Escola Nacional de Saúde Pública Sergio Arouca, Rio de Janeiro & Saúde Pública & 2 & - & $2(3)$ \\
\hline & & Bioética, Ética Aplicada e Saúde Coletiva & & & \\
\hline & Pontifícia Universidade Católica, Rio de Janeiro & Psicologia & - & 1 & $1(2)$ \\
\hline & Pontifícia Universidade Católica de São Paulo & Psicologia Clínica & 2 & - & $2(3)$ \\
\hline & Universidade Federal de Minas Gerais & Ciências da Saúde & 1 & - & 1(2) \\
\hline & Fundação Oswaldo Cruz & Ciências da Saúde & - & 1 & $1(2)$ \\
\hline & Universidade Estadual de Campinas & Ciências Médicas & 1 & - & $1(2)$ \\
\hline $\begin{array}{l}\text { Centro- } \\
\text { oeste }\end{array}$ & Universidade Federal de Goiás & Música & 1 & - & 1(2) \\
\hline Total & 25 & 35 & 47 & 13 & $60(100)$ \\
\hline
\end{tabular}




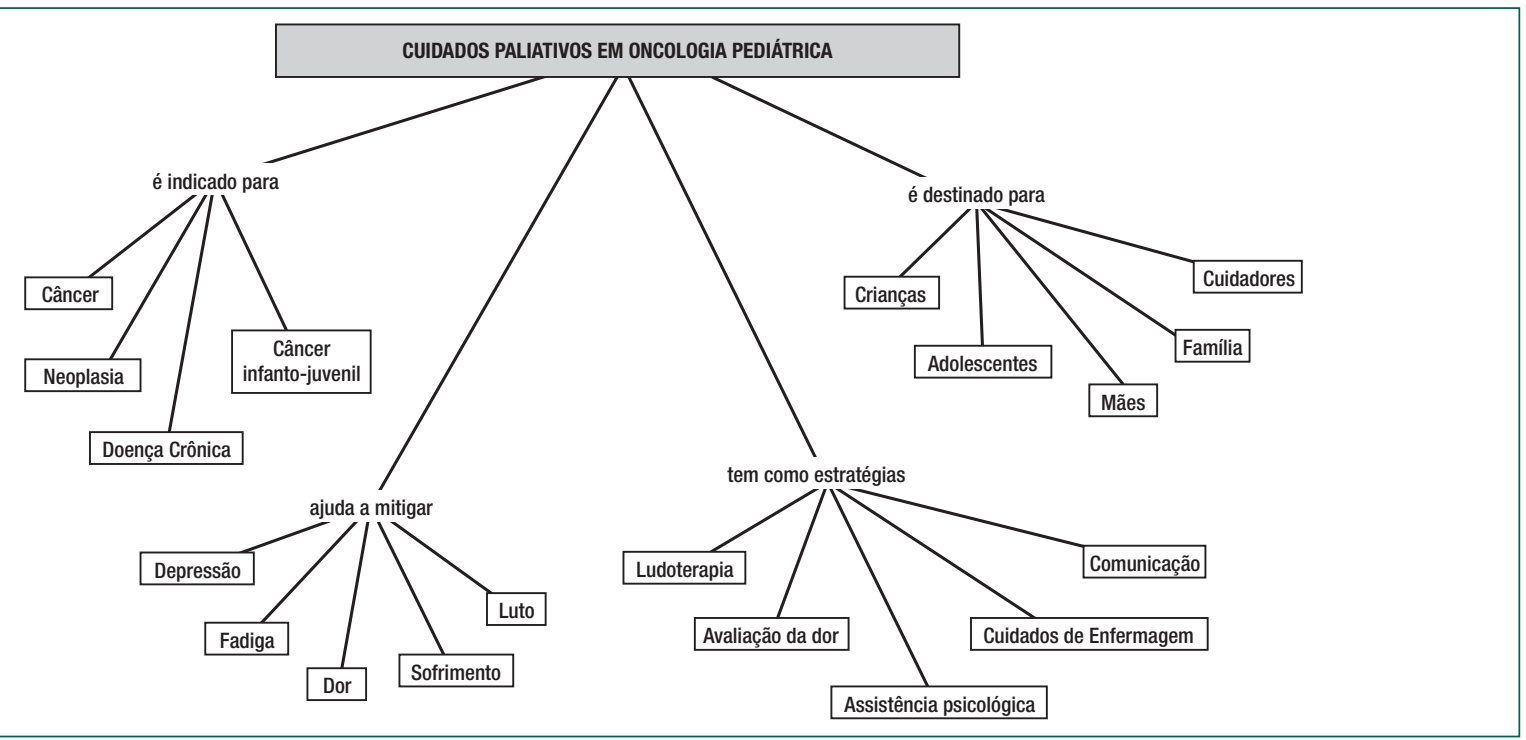

Figura 2. Mapa conceitual elaborado a partir das palavras-chave das dissertações e teses selecionadas para 0 estudo

Foram identificados 35 Programas de PósGraduação, desse total, os Programas da Universidade de São Paulo (USP) campus de Ribeirão Preto, produziu maior quantitativo de dissertaçóes e teses, apresentando um percentual 23\%, a saber: o Programa de Pós-Graduação em Psicologia, o Programa de Pós-Graduação em Enfermagem Psiquiátrica e com maior destaque para o Programa de Pós-Graduação em Enfermagem em Saúde Pública com nove (15\%) deste percentual. O Programa de Pós-Graduação em Enfermagem da Universidade Federal da Paraíba apresentou, no período em questão, seis (10\%), sendo quatro dissertaçóes de mestrado e duas teses de doutorado (Tabela 1).

Nos dados inerentes à formação profissional dos autores dos estudos, a Enfermagem destacou-se como a profissáo com o maior número de defesas com 29 estudos (48\%), a Psicologia com 19 trabalhos $(32 \%)$ a Medicina com sete estudos (12\%) e os seis restantes distribuídos entre as formaçóes profissóes de fisioterapia, filosofia, odontologia, bioética e musicoterapia ficando a distribuição de um (2\%) para cada. Quanto ao método de investigação adotado nos 60 estudos elegidos para a pesquisa, destacou-se a abordagem qualitativa em 49 estudos (82\%). Pesquisas de abordagem quantitativa corresponderam à sete estudos (12\%) e de abordagem quanti-qualitativa à quatro estudos (6\%). No tocante às palavras-chaves das teses e dissertaçóes que compuseram a amostra desta pesquisa, essas foram agrupadas, analisadas, e as mais evidenciadas possibilitaram a construção de um mapa conceitual apresentado na figura 2 .

\section{Discussão}

Os dados revelam que houve um crescimento nas pesquisas de dissertaçóes e teses a partir de 2011. Diante desse resultado, sabe-se que o avanço dos cuidados paliativos é uma realidade importante na Área da Saúde como um todo. Esse achado pode estar relacionado à publicação da Resolução CFM 1973/2011 do Conselho Federal de Medicina, criando, entre outras áreas de atuação, a medicina paliativa. Além disso, associam os cuidados paliativos às especialidades da clínica médica, como a pediatria. ${ }^{(7)}$

No tocante à concentração regional das produçóes, essa foi maior na Regiâo Sudeste, e a Região Norte com o menor destaque. Em relação ao Programa, destacou-se o Programa de PósGraduação em Enfermagem em Saúde Pública da Universidade de São Paulo, campus de Ribeirão Preto-SP. No que se refere à distribuição dos programas de pós-graduação no Brasil, no ano de 2017, último ano analisado pela Capes, foram 681 Programas de Pós-Graduação, na área de conheci- 
mento de Ciência da Saúde. Destes, a maioria se concentra na Região Sudeste. ${ }^{(8)}$

Pesquisa assinala que há uma grande desigualdade entre as regióes, e o motivo é gerado pela distribuição de recursos. O Sudeste recebeu do $\mathrm{CNPq}$ mais de $50 \%$ de recursos e apenas um pouco mais de $2 \%$ foi destinado à Região Norte do País, dados atualizados pelo portal Geocapes em 20 de agosto de 2018. Nessa conjuntura, é improvável diminuir as diferenças, e esse quadro continuará se perpetuando, resultando numa maior concentração de programas de pós-graduação, atingindo, também um maior percentual de mestres e doutores nas regiôes Sul-Sudeste. ${ }^{(9)}$

Dentre os profissionais de saúde que atuam em serviços de cuidados paliativos, destacou-se a equipe de enfermagem. Estudo aponta dados expressivos em relação à contribuição científica brasileira para a prática de enfermagem em cuidados paliativos a crianças e adolescentes com câncer, sendo o país com maior número de publicaçôes. ${ }^{(10)}$

Quanto ao desenho metodológico, a maioria dos estudos são de abordagem qualitativa. Estudo qualitativos buscam a investigação dos fenômenos, exploram a magnitude dos acontecimentos, preocupa-se com as singularidades e significados. ${ }^{(10)}$

Em relação aos artigos originados das dissertaçóes e teses, observou-se um importante indicador biliométrico em relação a dissertação intitulada "Cuidados paliativos: relação dialógica entre enfermeiros e criança com câncer" que divulgou seus achados em três artigos publicados em periódicos divulgação científica de enfermagem, saúde e de outras áreas afins, a saber: Importância da comunicação nos cuidados paliativos em oncologia pediátrica: enfoque na Teoria Humanística de Enfermagem, Revista Latino-Americana de Enfermagem, ano de publicação 2013; Cuidados paliativos à criança com câncer, Revista Enfermagem UERJ, ano 2013; Vivência de enfermeiros no cuidado à criança em fase terminal: estudo à luz da teoria humanística de enfermagem, no periódico - Revista Ciência, Cuidado e Saúde, ano 2014.

A dissertação intitulada "Benefícios da ludoterapia como cuidado paliativo em crianças hospitalizadas com câncer” foi o estudo que apresentou o maior nível de evidência científica de acordo com estudiosos sobre a prática baseada em evidência por se tratar de uma pesquisa quantitativa com desenho analítico experimental e delineamento de ensaio clínico não randomizado. ${ }^{(1)}$ Portanto, é classificada no nível 3: evidências obtidas de ensaios clínicos bem delineados sem randomização. A categorização do nível de evidência foi baseada no EvidenceBasedPractice in NursingandHealthcare. ${ }^{(12)}$

Quanto ao cenário onde as pesquisas foram realizadas o ambiente hospitalar foi o mais prevalente, contabilizando $98 \%$ das pesquisas, sendo $1 \%$ de publicação em uma Instituição Filantrópica de apoio á criança com câncer e 1\% com profissionais do município de Criciúma - Santa Catarina que trabalha com crianças e adolescentes na terminalidade. No que concerne aos estados brasileiros, todos possuem pelo menos um hospital habilitado em oncologia, onde o paciente oncológico se deparará desde a realização de um exame até cirurgias mais complexas, contabilizando um quântico de 317 entre unidades e centros de assistência habilitados no tratamento do câncer. ${ }^{(13)}$

Ainda de acordo com o a pesquisa, o modelo de atendimento prevalente é o do tipo ambulatorial (53\%), que atende a pacientes com câncer e com outras patologias ameaçadoras da vida. A prevalência da população assistida nos estudo é de adultos (88\%) e de idosos (84\%), com financiamento público. ${ }^{(13)}$

No que diz respeito aos serviços consolidados em cuidados paliativos, estudo assinala que 50\% dos 177 serviços brasileiros de cuidados paliativos atuam no estado de São Paulo. Apenas 13 serviços atuam nas Regióes Norte e Nordeste do País. ${ }^{(14)}$

O método de mapeamento de conceitos é considerado uma ferramenta de organização e representação do conhecimento relacionado entre conceitos que facilitam a reflexão estratégica. São também denominados de unidades semânticas ou unidades de sentido. No mapa conceitual (Figura 2), as palavras-chave mais frequentes foram organizadas em quatro eixos referentes à temática: cuidados paliativos e oncologia pediátrica. ${ }^{(15)}$

O primeiro e o segundo eixos semânticos deste estudo dizem respeito à indicação e a quem são destinados os cuidados paliativos, sendo estes cuidados 
indicados para todos os pacientes (idosos, adulto, crianças e adolescentes), com doença ameaçadora da continuidade da vida. Pode ser principiado junto à terapêutica curativa e quanto mais precoce o início da paliação, melhor para a promoção da qualidade de vida para os envolvidos nesse processo. ${ }^{(16)}$

Este estudo demonstrou que os cuidados paliativos em oncologia pediátrica são indicados para tratar o câncer infanto-juvenil e doenças crônicas, abrangendo também mães, cuidadores e familiares destes. Durante a evoluçáo da doença, a intensidade desses cuidados varia, uma vez que o foco e os objetivos vão, progressivamente, transitando de uma ênfase em tratamentos modificadores da doença até abordagens com intenção exclusivamente paliativas que vão impactar, principalmente, na qualidade de vida. ${ }^{(17)}$

O terceiro eixo diz respeito ao controle dos sintomas, como a dor, fadiga, sofrimento psíquico e o luto. Na atenção paliativa, o enfoque terapêutico visa ao alívio dos sintomas que comprometem a qualidade de vida, integrando ações interdisciplinares e interprofissionais, cujas dedicaçóes se quantificarão em função das necessidades concretas de cuidado.

O desafio da equipe paliativa é cuidar do ser humano na sua totalidade, exercendo uma ação preferencial em relação à sua dor e ao seu sofrimento biopsicossocial e espiritual, com capacidade científica e técnica, além da sensibilidade ao sofrimento do outro, o que pode facilitar o cuidado integral e humanizado. ${ }^{(18)}$ Cabe salientar que o cuidado interdisciplinar é um elemento central do tratamento do câncer e fornece uma base sólida para incorporar elementos adicionais de cuidados paliativos à oncologia pediátrica. ${ }^{(19)} \mathrm{O}$ quarto e último eixo do mapa conceitual faz relação à comunicação como estratégia nos cuidados paliativos em oncologia pediátrica, relaciona-se também à ludoterapia, à psicoterapia e à assistência de enfermagem.

Ressalta-se que a comunicação entre a equipe de cuidados paliativos, paciente e seus familiares afeta a experiência da doença e o plano assistencial, assim como a satisfação das pessoas envolvidas nesse cuidado. A linguagem deve ser clara e simples, se necessário recorrendo a materiais lúdicos para facilitar a compreensão, procurando dar apoio ao paciente, fazendo uso de habilidades de comunicação que permitam minimizar o impacto emocional que as notícias difíceis suscitam. ${ }^{(20)}$

Observa-se que cuidado à criança é complexo, o que exige dos profissionais, além de conhecimento técnico-científico, capacidade emocional para ajudar às famílias a enfrentarem a fase de final de vida, inclusive no período do luto. ${ }^{(21)}$ Assim sendo, precisa que esse processo de enlutamento seja discutido, a fim de aprimorar o apoio oferecido aos envolvidos (criança e família), visto que se encontram fragilizados diante de uma situação difícil. Todos necessitam de acolhimento, respeito e cuidado às suas especificidades. ${ }^{(22)}$

Em relação à assistência de enfermagem em cuidados paliativos, estudo ressalta que o enfermeiro é o profissional que está diretamente ligado ao paciente, desde o diagnóstico de uma doença que ameaça a vida até a sua finitude. ${ }^{(23)}$ Por essa razão, tem o dever de escutar e de compreender bem mais as necessidades dos pacientes, dando-lhes apoio no momento de angústia ao enfrentar uma doença que ameaça a continuidade da vida. ${ }^{(24)}$ Estudo aponta que profissionais da equipe de cuidados paliativos buscam compartilhar diversos conhecimentos com o intuito de estabelecer as estruturas desse cuidado singular, em que a troca de saberes e de experiências os ajuda a planejar suas açóes diante dessa perspectiva. ${ }^{(24)}$ Outro aspecto que merece destaque é a elaboração do planejamento terapêutico individualizado que tem a proposta de inserir a família e valorizar a criança com câncer como integrantes essenciais do cuidado. ${ }^{(25)}$

Vale ressaltar que a promoção de cuidados paliativos é de fundamental importância para a criança com doença que ameaça a continuidade da vida, como o câncer. Portanto, profissionais da área da saúde, em particular o enfermeiro, devem propiciar uma assistência integral que vise à melhoria da qualidade de vida da criança, como também procurem apoiar a sua família no enfrentamento das situaçôes difíceis durante todo o tratamento até o período do luto.

\section{Conclusão}

A pesquisa identificou um número reduzido de estudos oriundos de dissertaçóes e teses, sobre cuidados paliativos em oncologia pediátrica, defendidas 
em Programas de Pós-Graduação no cenário brasileiro. Contudo, o indicador bibliométrico pertinente às palavras-chave demonstra que há evidências de que a referida temática encontra-se em desenvolvimento, visto que o quantitativo de eixos temáticos apresentados no mapa conceitual destaca a variedade de sua abrangência. Sugerem-se novos estudos para ampliar a produção científica acerca do tema, bem como para disseminar evidências científicas no contexto da prática clínica dos cuidados paliativos em oncologia pediátrica.

\section{Colaborações}

Dias KCCO, Batista PSS, Fernandes MA, Zaccara AAL, Oliveira TC, Vasconcelos MF, Oliveira AMM e Andrade FF declaram que contribuíram com a concepção do estudo, análise e interpretação dos dados, redação do artigo e aprovação da versão final a ser publicada.

\section{Referências}

1. Instituto Nacional de Câncer (INCA). Estimativas 2018: incidência de câncer no Brasil. Rio de Janeiro: INCA; 2017. 130 p.

2. Academia Nacional de Cuidados Paliativos (ANCP). Análise situacional e recomendações para estruturação de programas de cuidados paliativos no Brasil. [Internet]. Brasília (DF): ANCP; 2018 [citado 2019 Jun 10]. Disponível em: https://paliativo.org.br/wp-content/ uploads/2018/12/ANALISE-SITUACIONAL_ANCP-18122018.pdf

3. Misko MD, dos Santos MR, Ichikawa CR, de Lima RA, Bousso RS. The family's experience of the child and/or teenager in palliative care: fluctuating between hope and hopelessness in a world changed by losses. Rev Lat Am Enfermagem. 2015;23(3):560-7.

4. Friedrichsdorf SJ, Postier A, Dreyfus J, Osenga K, Sencer S, Wolfe J. Improved quality of life at end of life related to home-based palliative care in children with cancer. J Palliat Med. 2015;18(2):143-50.

5. Galvão TF, Pansani TS, Harrad D. Principais itens para relatar revisões sistemáticas e meta-análises: a recomendação PRISMA. Epidemiol Serv Saude. 2015;24(2):335-42.

6. Alves AM, Costa SF, Fernandes MA, Batista PS, Lopes ME, Zaccara AA. Communication in palliativecare: a bibliometricstudy. J Res Fundam Care Online. 2019;11(2):524.

7. Conselho Federal de Medicina. (CFM). Resolução $n^{0}$ 1.973/2011. Brasília (DF): CFM; 2011. Seção I, p. 144-7.
8. Coordenação de Aperfeiçoamento de Pessoal de Nível Superior (Capes). GeoCapes [Internet]. Brasília (DF): Capes; 2019. [citado 2019 Jun 12]. Disponível em: https://geocapes.capes.gov.br/geocapes/

9. Sousa AD, Silva LF, Paiva ED. Nursing interventions in palliative care in Pediatric Oncology: an integrative review. Rev Bras Enferm. 2019;72(2):531-40.

10. Minayo CS. Amostragem e saturação em pesquisa qualitativa: consensos e controvérsias. RevPesquiQuali. 2017;5(7):1-12.

11. Silva F. Benefícios da ludoterapia como cuidado paliativo em crianças hospitalizadas com câncer. Maranhão [dissertação]Recife: Universidade Federal do Maranhão; 2009.

12. Pedrosa KK, Oliveira IC, Feijão AR, Machado RC. Enfermagem baseada em evidência: caracterização dos estudos no Brasil. Cogitare Enferm. 2015;20(4):733-41.

13. Instituto Nacional de Câncer (INCA). Onde tratar pelo SUS. Rio de Janeiro: INCA; 2019. p. 568-83.

14. World Health Organization (WHO). Global atlas of palliative care at the end of life 2014. Geneva: WHO; 2017. 103 pp.

15. Bittencourt GK, da Nóbrega MM, Medeiros AC, Furtado LG. Mapas conceituais no ensino de pós-graduação em enfermagem: relato de experiência. Rev Gaúcha Enferm. 2013;34(2):172-6.

16. World Health Organization (WHO). Palliative Care Fact Sheet. Geneva: WHO; 2018.

17. Macedo A, Mercês NA, Silva LA, Sousa GC. Estrategias de enfrentamiento de losprofesionales de enfermería frente alamuerte em la oncologia pediátrica: revisión integrativa. Rev Pesq Cuid Fundam. 2019;11(3):718-24.

18. Downing J, Boucher S, Daniels A, Nkosi B. Pediatric palliative care in resource-poor countries. Children (Basel). 2018;5(2):27.

19. Cervantes BJ, Jones $E$. The interdisciplinary oncology team and the role of palliative care consultation. In: Wolfe J, Jones B, Kreicbergs $\mathrm{U}$, Jankovic $\mathrm{M}$, editors. Palliative care in pediatric oncology. Pediatr Oncology; 2018. https://doi.org/10.1007/978-3-319-61391-8_3.

20. Szymczak JE, Schall T, Hill DL, Walter JK, Parikh S, DiDomenico C, et al. Pediatric oncology providers' perceptions of a palliative care service: the influence of emotional esteem and emotional labor. J Pain Symptom Manage. 2018;55(5):1260-8.

21. Silva AF, Issi HB, Motta MG, Botene DZ. Cuidados paliativos em oncologia pediátrica: percepções, saberes e práticas na perspectiva da equipe multiprofissional. Rev Gaúcha Enferm. 2015;36(2):5662.

22. Andrade ML, Mishima GF, Barbieri V. Recriando a vida: 0 luto das mães e a experiência materna. Psicol Teor Prat. 2017;19(1):21-32.

23. Sousa JM, Alves ED. Nursing competencies for palliative care in home care. Acta Paul Enferm. 2015;28(3):264-9.

24. Fernandes MA, Evangelista CB, Platel IC, Agra G, Lopes MS, Rodrigues FA. Percepção dos enfermeiros sobre o significado dos cuidados paliativos em pacientes com câncer terminal. Ciênc Saúde Coletiva. 2013;18(9):2589-96.

25. Johnston DL, Nagel K, Friedman DL, Meza JL, Hurwitz CA, Friebert $S$. Availability and use of palliative care and end-of-life services for pediatric oncology patients. J Clin Oncol. 2008;26(28):4646-50. 